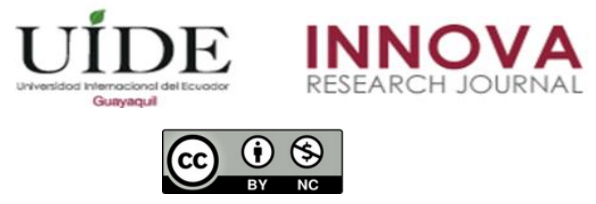

INNOVA Research Journal, ISSN 2477-9024

(Septiembre-Diciembre 2019). Vol. 4, No.3.1 pp. 72-88

DOI: https://doi.org/10.33890/innova.v4.n3.1.2019.1042

URL: http://revistas.uide.edu.ec/index.php/innova/index

Correo: innova@uide.edu.ec

\title{
El uso de las TICS en colegios públicos de la ciudad de Loja
}

\section{The use of ICTs in public schools in the city of Loja}

Silvia Alexandra Jaramillo Luzuriaga

Lorena del Cisne Tacuri Peña

Celso Dario Hurtado Cuenca

Carmen Paulina Muñoz Cabrera

Universidad Internacional del Ecuador, Ecuador

Autor para correspondencia: sijaramillolu@uide.edu.ec; lotacuripe@uide.edu.ec; cehurtadocu@uide.edu.ec; cmunoz@uide.edu.ec

Fecha de recepción: 08 de julio de 2019 - Fecha de aceptación: 30 de septiembre de 2019

\section{Resumen}

El siguiente artículo tiene por objetivo analizar la importancia del uso de las técnicas de la información y la comunicación en los colegios públicos de la ciudad de Loja, como estrategia metodológica para mejorar el proceso de enseñanza aprendizaje, puesto que cada vez es más amplia la información que se puede compartir mediante los diferentes soportes tecnológicos. La metodología que se utilizó incluye todo un proceso investigativo enmarcado en los métodos inductivo-deductivo; además para obtener la información empírica sobre el objeto de estudio se aplicó encuestas a los directores de los colegios públicos de la ciudad de Loja. En los resultados obtenidos en la investigación se revelaron que las técnicas de la información y la comunicación se han convertido en una poderosa herramienta para el proceso de enseñanza aprendizaje, ya que es un medio muy utilizado tanto en la educación media como en todos los ámbitos educativos; ello demuestra principalmente que la utilización de las técnicas de comunicación abre la posibilidad de aumentar el nivel de participación, la comunicación y la socialización del conocimiento con el afán de lograr aprendizajes significativos por medio del uso de recursos tecnológicos.

Palabras claves: técnicas de la información y la comunicación; recursos tecnológicos; docentes; proceso; enseñanza-aprendizaje

\begin{abstract}
The following article aims to analyse the importance of the use of information and communication techniques in public schools in the city of Loja, as methodological strategy to improve the teaching-learning process, since it is increasingly more extensive information that can be shared by different technological supports. The methodology used includes an entire investigative process framed in inductive-deductive methods; In addition the empirical information about the object of study applied surveys to principals of public schools in the city of Loja. In the results of the research were revealed that information and communication techniques have become a powerful tool for the teaching-learning process, since it is a widely used medium in both secondary education and all educational levels; It mainly demonstrates that the use of the techniques of communication opens the possibility of increasing the level of participation, communication and
\end{abstract}


socialization of knowledge in an effort to achieve meaningful learning through the use of technological resources.

Key words: information and communication techniques; technological resources; teachers; process; teaching-learning

\section{Introducción}

En la actualidad el uso de las tecnologías de la información en los centros educativos es fundamental para el desarrollo del aprendizaje, donde los alumnos pueden investigar y aplicar sus conocimientos de manera integral, en este caso los colegios públicos contribuyen a fomentar la construcción del conocimiento y la creación de un ambiente positivo para la enseñanza y el aprendizaje.

Sn embargo en algunos colegios públicos de la ciudad de Loja, no existe un adecuado ambiente de aprendizaje que facilite su enseñanza mediante el uso de recursos, experiencias, conceptos, actitudes y destrezas que les permitan desarrollar un panorama claro en la búsqueda del conocimiento y en su crecimiento personal. Así como la falta de recursos tecnológicos, tales como: videos, folletos electrónicos, bibliotecas virtuales, programas (software) y otros, por medio del computador y el cyber espacio.

En este artículo el tema principal es el uso de las TICS en los colegios públicos de la ciudad de Loja, los avances en las técnicas de información y comunicación han transformado de manera general la forma en que la sociedad se desenvuelve. Por tanto, es importante su estudio dentro de la educación y los procesos de enseñanza aprendizaje que se ven influenciados por la innovación tecnológica a la que se encuentran expuestos generando en este contexto la oportunidad de desarrollar nuevas estrategias de enseñanza aprendizaje, incorporando herramientas tecnológicas disponibles en los centros educativos, lo que brinda la posibilidad de aumentar el nivel de participación, la comunicación y la socialización del conocimiento con el fin de lograr aprendizajes significativos por medio del uso de recursos tecnológicos disponibles.

En este sentido, el presente artículo surge ante la necesidad de conocer como las técnicas de la información y comunicación como estrategia metodológica potencian el proceso de enseñanza aprendizaje de los alumnos de los colegios públicos de la ciudad de Loja, para ello fue preciso adoptar una metodología cimentada en el uso de métodos y técnicas de investigación.

Por consiguiente, con este artículo se busca identificar la necesidad e importancia del uso de las TICS como un elemento tecnológico necesario, ya que si se aplican correctamente tiene muchas ventajas como mejora de las herramientas, elementos, habilidades, conocimientos y destrezas que desarrollan los usuarios al establecer proyectos de manera virtual o en línea. Es decir, cuando la tecnología se aplica en la enseñanza y aprendizaje posibilita grandes oportunidades para generar nuevos ambientes de aprendizaje donde los alumnos siendo los entes principales logran aprendizajes significativos acordes al medio en que se desenvuelven. 


\section{Marco teórico}

\section{¿Qué son las TICS?}

Las tecnologías de la información y comunicación TICS, se conciben como el universo de dos conjuntos, representados por las tradicionales tecnologías de la comunicación TC, constituidas principalmente por la radio, la televisión y la telefonía convencional; y por las tecnologías de la Información TI, que se encargan del diseño, desarrollo, fomento, mantenimiento y administración de la información por medio de sistemas informáticos, para información, comunicación o ambos (Samillán, 2010).

Originariamente, la Tecnología de la Educación se presentó como una disciplina cuyo objetivo fundamental se centraba en el estudio de los instrumentos y equipos técnicos y sus distintas formas de utilización escolar, considerados estos instrumentos como vehículo o soporte de diversas funciones didácticas, especialmente, la presentación de estímulos y contenidos a los estudiantes. La base de esta tecnología estaba constituida por los medios audiovisuales: cine, imagen fija, registro de sonido, radio, etc. que se habían venido desarrollando desde finales de la 2a guerra mundial (Bautista \& Alba, 1997).

Peralta (2002) define las TICS como "aquellas herramientas computacionales e informáticas que procesan, sintetizan, recuperan y presentan información representada de la más variada forma" (pág. 88). Por tanto, se trata de un conjunto de herramientas, soportes y canales para el tratamiento y acceso a la información, para dar forma, registrar, almacenar y difundir contenidos digitalizados.

\section{Componentes de las TICS}

Tomando en cuenta que vienen de la unión de TI y TC, se diferencian sus componentes de la siguiente manera: 


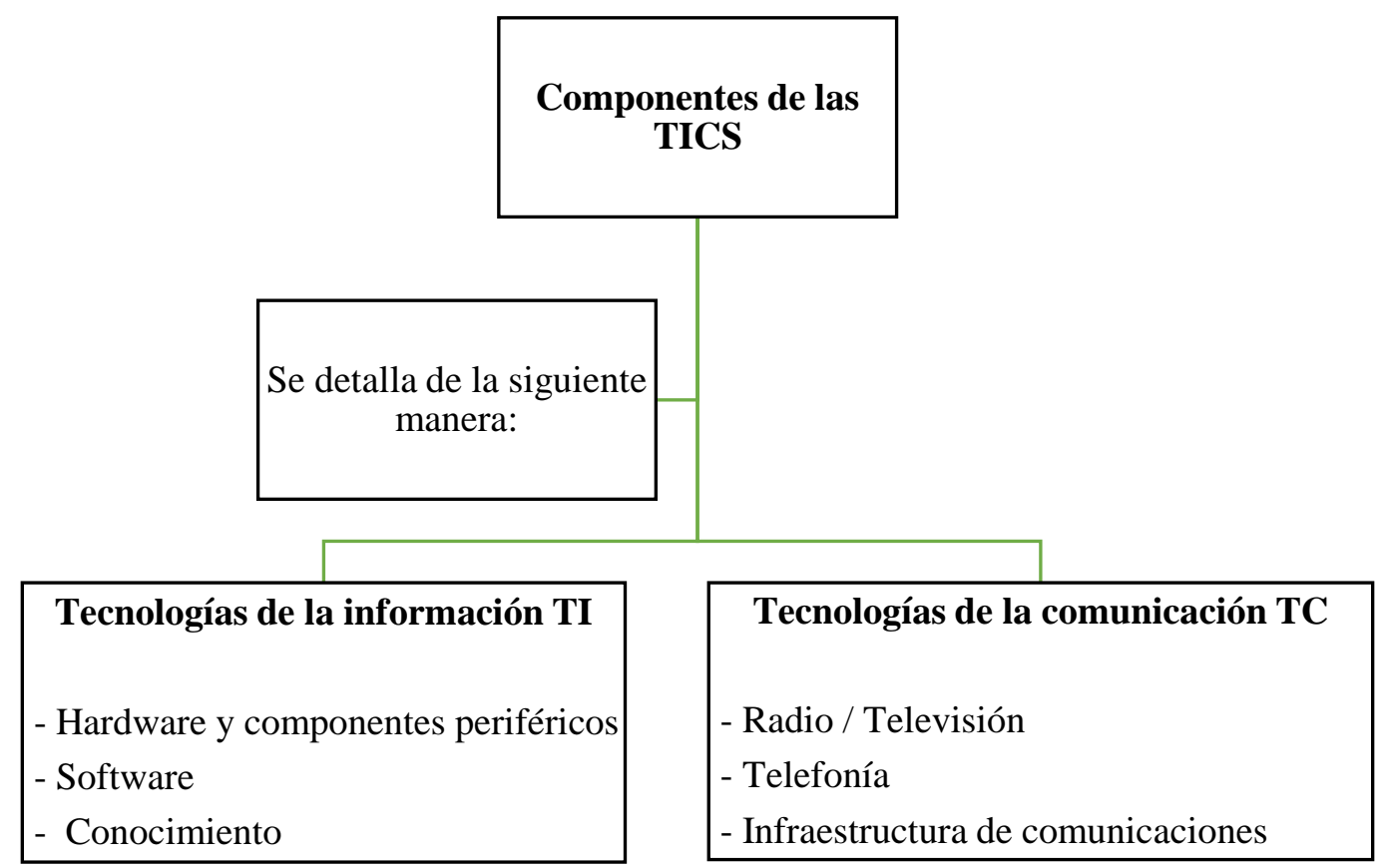

Figura 1. Componentes de las TICS

Fuente: (Jaramillo \& González, 2016).

Las TICS son herramientas que permiten la adquisición, producción, almacenamiento, tratamiento, comunicación, registro y presentación de informaciones, en forma de voz, imágenes y datos contenidos en señales de naturaleza acústica, óptica o electromagnética (Jaramillo \& González, 2016).

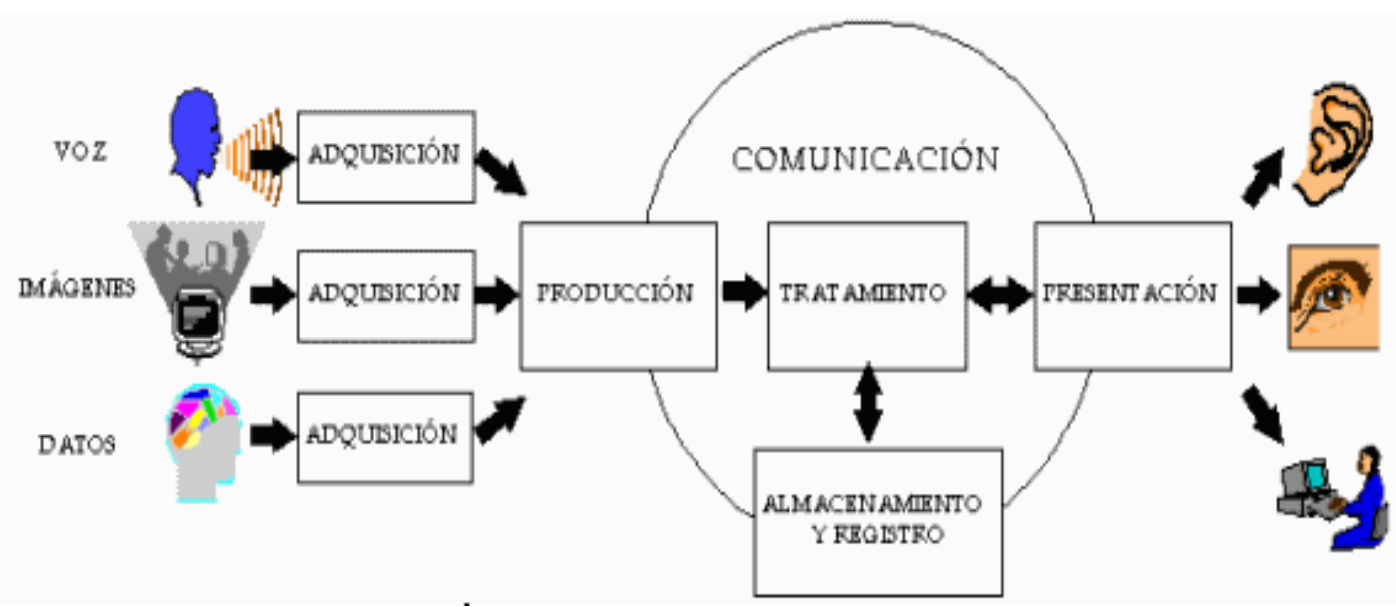

Figura 2. El proceso de la información

Fuente: http://www.monografias.com/trabajos37/tic-en-educacion/Image7932.gif

\section{Las TICS y la formación académica}

En la actualidad el protagonismo de las tecnologías de la información y las comunicaciones es cada vez mayor, esto ya sea para nuestra cotidianidad como para aspectos más complejos, como puede ser un contexto educativo. Internet es una herramienta de uso 
masivo que permite a las personas acceder a conocimiento e información de forma casi inmediata y a la carta de quien busque, así, se transforma en una fuente inagotable de datos e información, de la cual no entraremos en cuestionamiento de veracidad, más de accesibilidad y de potencial uso (Fabres, Libuy, \& Tapia, 2014).

Ramírez (2009) en la formación académica define el proceso de enseñanza como "la transmisión de una serie de conocimientos, técnicas, normas, y/o habilidades. Está basado en diversos métodos, realizado a través de una serie de instituciones, y con el apoyo de una serie de materiales" (pág. 61).

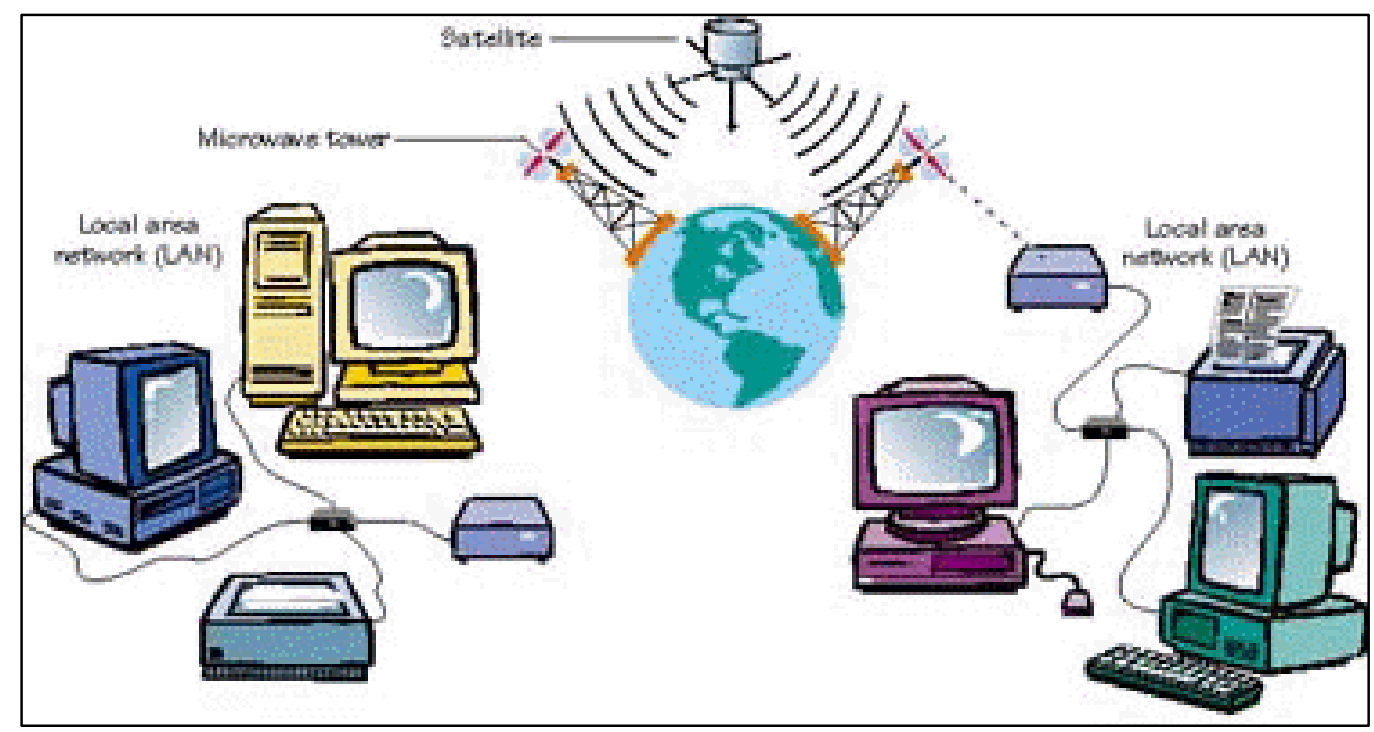

Figura 3. Internet

Fuente: http://www.hipertexto.info/images/SatLAN.gif

Estos últimos dos factores parecen ser los más relevantes a entender cómo pueden aportar al desarrollo de herramientas útiles en un contexto educacional. Así, hoy podemos encontrar cursos online o bien campus virtuales que permiten acceder a esta información de forma más específica y, en algunos casos, de forma más segura y confiable. La pregunta debiese ser entonces si este torrente de información disponible para todos se puede usar de forma tal que sea un aporte real al proceso de enseñanza y aprendizaje.

Las TICS favorecen el intercambio internacional de experiencia, lo que conlleva a una disminución de las barreras y distancias geográficas, enriqueciendo el aprendizaje individual y colectivo (Dussel \& Quevedo, 2010). Bajo ese contexto es válido cuestionarse el si los establecimientos educacionales están creando y/o compartiendo información educativa.

Pérez (2002), expresa que las TICS son las herramientas necesarias para acceder y manipular datos digitales, y explicita tres características básicas de las TICS: 


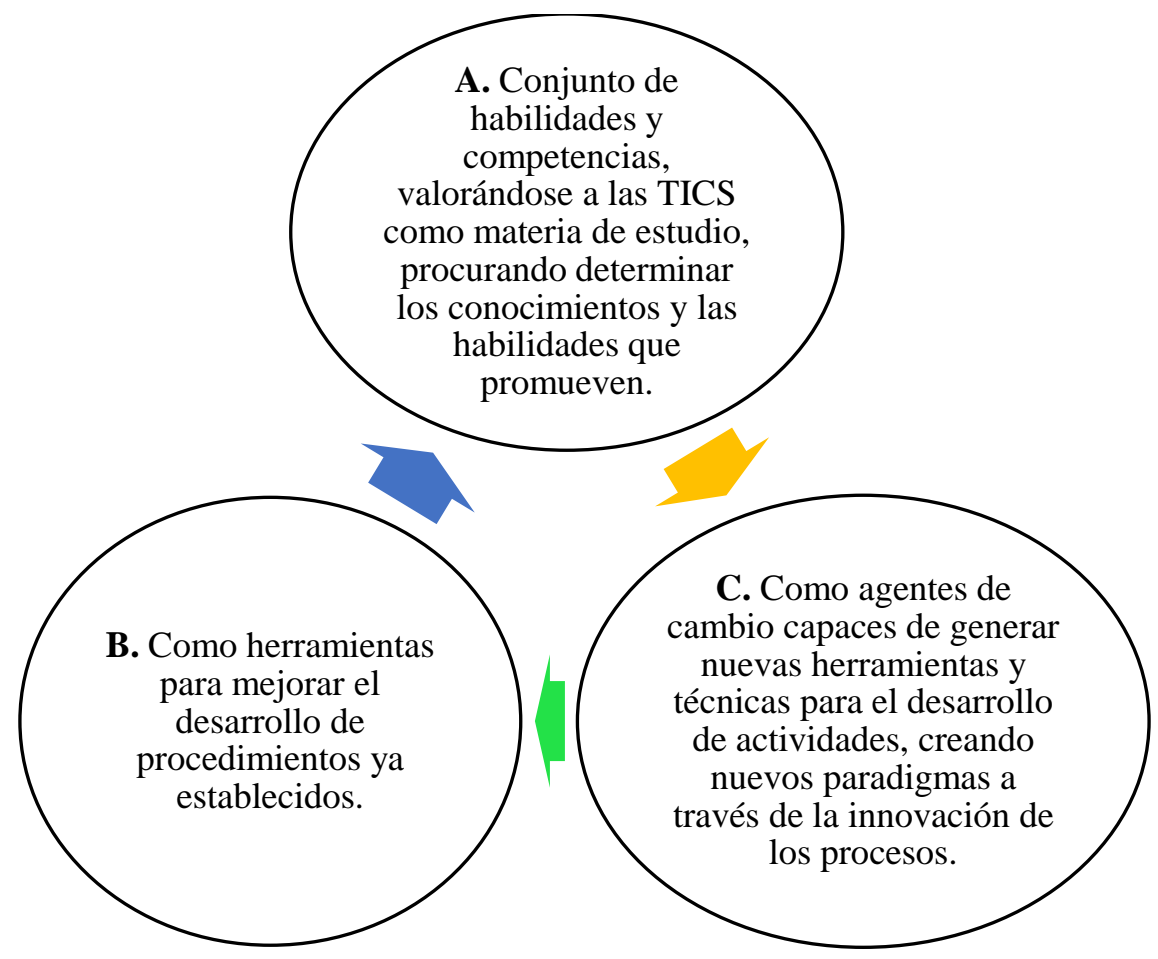

Figura 4. Características básicas de las TICS

Fuente: (Pérez, 2002)

\section{Las TICS en los establecimientos educacionales secundarios}

Las TICS son de gran utilidad para los procesos educativos y de enseñanza, generando transformaciones en la forma en que la educación se imparte y conoce. En la actualidad este nuevo escenario exige de la participación de los establecimientos educacionales y de quienes lo regulan, en donde, para empezar, se deben cambiar paradigmas que permitan realizar un cambio que parta desde los cimientos de la educación, creando una nueva que permitan tanto al docente como al estudiante vivir una transición controlada y supervisada (Fabres, Libuy, \& Tapia, 2014).

El desafío de esta transición surge de cómo estas tecnologías permiten innovar el proceso de aprendizaje en sí, y no aferrarse de la tecnología por sí misma. Esto quiere decir: innovar a través de su uso. Anteriormente se comenta que las tecnologías pueden moldearse para un usuario en particular, asimismo el usuario puede moldear las tecnologías. Ese es entonces el punto al cual los establecimientos debiesen apuntar; cómo sus propias necesidades moldean un sistema de información que les permita mejorar el proceso de enseñanza y cómo las tecnologías por si mismas pueden integrarse dada su naturaleza universal.

La incorporación de las TICS ha significado que para los espacios educativos estas dejen de ser una opción, y los esfuerzos de los países e instituciones se encaminen en la generación e implementación de iniciativas que impliquen el máximo aprovechamiento de las tecnologías en los procesos formativos (Severin, 2010). 


\section{Ventajas y desventajas de las TICS}

Hilarión (2014), expresa que es pertinente identificar las ventajas en la incorporación de las TICS por parte de los docentes, debido a la importancia de estos recursos en el proceso de enseñanza - aprendizaje, pero se hace necesario también explicar las desventajas de no usarlas dado a los avances y la rapidez con que cada día se hacen operativos los desarrollo en los entornos educativos. En este sentido este es el primer factor que afecta a los docentes, pues como menciona Nieto (2006) al respecto de las TICS la velocidad de su desarrollo es vertiginosa y la novedad que trae consigo también suscita temores, resistencias y luchas entre sus afiliados y detractores; para tener una idea más clara, los educadores pueden sentir dudas al apropiarse o rechazar estos cambios de manera definitiva, ya que por seguir en un paradigma tradicional se niegan la posibilidad de explorar herramientas tecnológicas en su quehacer, también por desconocimiento e incluso por prejuicios, dejando de lado la innovación de las estrategias pedagógicas, lo anterior, permite evidenciar la falta de capacitación e incluso de iniciativa para la implementación de estos recursos.

En ocasiones las clases tradicionales pueden funcionar y cumplir con el propósito que se definió, pero por otro lado, hay poblaciones de estudiantes que requieren otro tipo de metodologías, pues su comprensión es mejor a partir de estrategias novedosas y porque no decir que se puede lograr a partir de la implementación de las TICS, y es en este punto donde el docente debe pensar en innovar su quehacer pedagógico y hacer uso de nuevas herramientas o recursos para no quedarse en un paradigma tradicional, pero tampoco se puede caer en el error de pensar, que porque un aula cuenta con diferentes medios tecnológicos ya se está haciendo buen uso de ellos (Moreno, 2011).

Según Vera (2012) menciona algunas ventajas y desventajas desde la perspectiva del aprendizaje: 


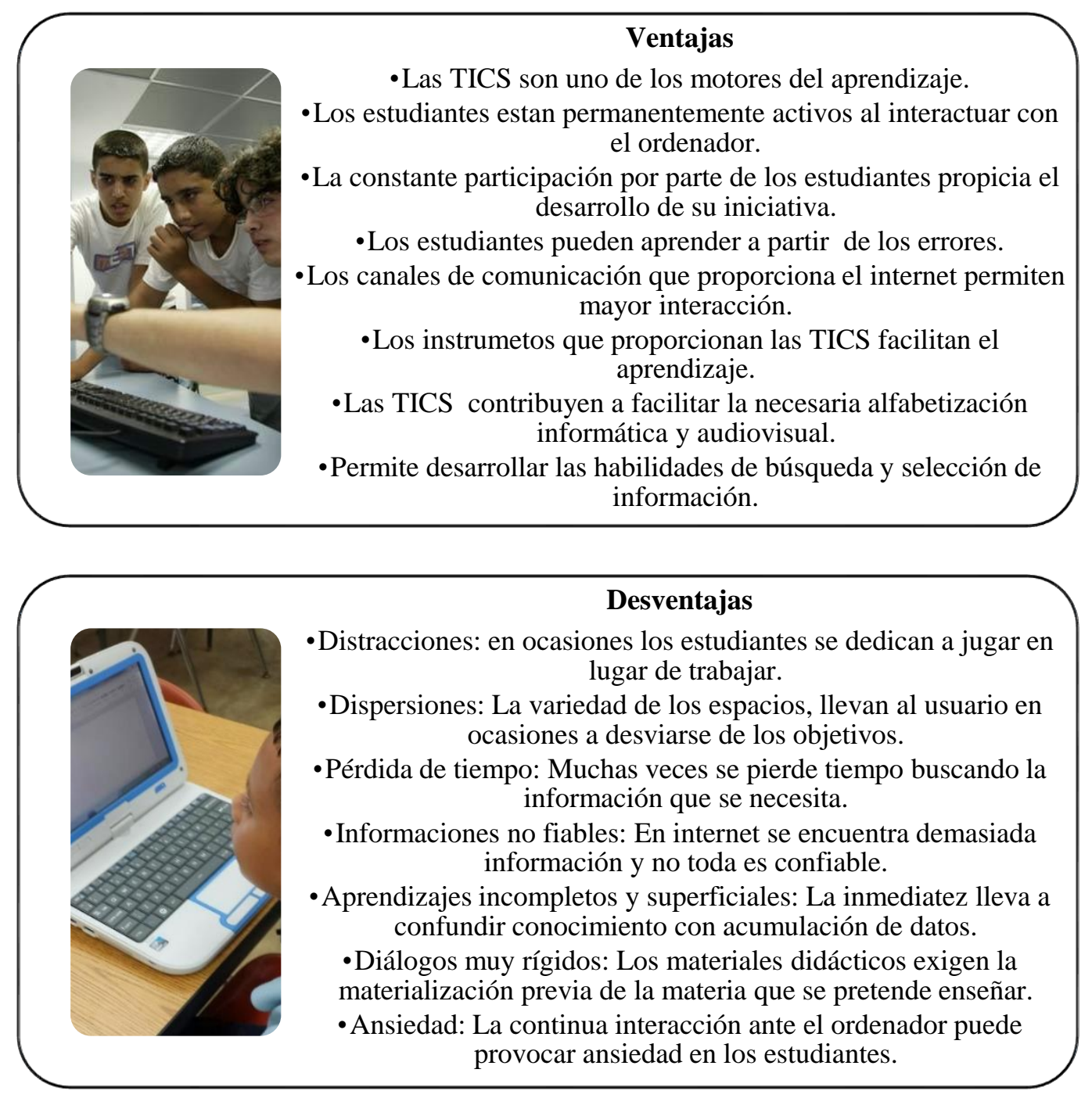

Figura 5. Ventajas y desventajas de las TICS

Fuente: (Vera, 2012)

A nivel mundial el acceso a las tecnologías, información y comunicaciones es indispensable en el campo de la educación moderna, donde ha ido incrementándose su uso como una herramienta básica para desarrollar con éxito los procesos de enseñanza de la sociedad contemporánea, a la cuál le permite actualizarse, recopilar información, así como fomentar el aprendizaje práctico, creativo, investigativo en niños estudiantes (Ramos, 2015).

El ingreso de las TICS en los procesos de enseñanza favorece la labor educativa poniendo a disposición de profesores y estudiantes gran cantidad de información a través de diversos canales que favorecen el trabajo colaborativo a través de entornos virtuales permitiendo la comunicación e interacción entre varios usuarios, y el fortalecimiento de destrezas y habilidades en el proceso de aprendizaje (Fernández \& Cebreiro, 2003). 


\section{Materiales y métodos}

\section{Diseño de la investigación}

Para la presente investigación se tomó en consideración una investigación de tipo cualitativa y cuantitativa; en donde el enfoque cuantitativo hace mención al estudio a partir del análisis de cantidades. En cambio, la investigación cualitativa tiene que ver con la exploración no numérica de datos, es decir se relaciona más con la interpretación inductiva todo ello para obtener información de confiabilidad y validez. Tomando en cuenta que este artículo está basado en conocer el uso de las técnicas de la información y la comunicación en los colegios públicos de la ciudad de Loja como apoyo a la educación; lo primordial fue poder conocer qué tipo de técnicas de la información y la comunicación se están implementando actualmente en los colegios, cuál es la disposición de los mismos por aplicarlas, y si efectivamente se les está dando el uso completo y eficiente que éstas pueden entregar.

Además, de acuerdo a las características y a la naturaleza del objeto de investigación, este trabajo revierte el carácter de no experimental, puesto que no existieron posibilidades de manipular las variables; la función del investigador consistió en la descripción de la realidad, tal como se presentó, para de esta forma, realizar un análisis crítico y poder plantear las alternativas de mejora que constan en la propuesta.

\section{Métodos de investigación}

Como métodos de investigación se utilizó los siguientes:

\section{- Método inductivo:}

Este método se lo aplicó en el análisis e interpretación de la encuesta a los directores, para la obtención de datos primarios, y realizar el análisis de las características de los procesos de enseñanza aprendizaje que se desarrollan en los colegios públicos de la ciudad de Loja, sobre los procesos de utilización de las técnicas de información y comunicación.

\section{- Método deductivo:}

Este método sirvió para la recopilación de conceptos teóricos y dar forma al marco teórico con contenidos precisos para el desarrollo de la investigación, así también permitió relacionar el problema objeto de estudio desde lo general a lo concreto o particular, específicamente en lo relacionado a los procesos de utilización de las técnicas de información y comunicación permitiendo la construcción de los lineamientos alternativos.

\section{Técnicas e instrumentos de investigación}

Entre las técnicas de investigación se utilizó los siguientes: 


\section{- Encuesta:}

Para obtener información empírica sobre el objeto de estudio se utilizó la encuesta. El cuestionario, técnicamente diseñado, se concibió con el propósito de obtener información sobre las características de los procesos de interactividad que se llevan a cabo en los alumnos. Así como recabar información referida a los aprendizajes significativos.

Este instrumento se aplicó a los directores de los colegios públicos de la ciudad de Loja, a través de un cuestionario previamente elaborado, el mismo que incluyó preguntas específicas sobre el tema a investigar. Para su ejecución fue necesario la organización y ejecución de una reunión de trabajo con los directores de los colegios de bachillerato, para explicar los propósitos de la investigación y la intencionalidad de la encuesta, de tal manera que ésta se desarrolle en un ambiente de tranquilidad y confianza, sin interrumpir las actividades académicas planificadas, $\mathrm{y}$ de esta forma obtener información relevante.

\section{Población y tamaño de la muestra}

La población en este artículo se consideró los colegios públicos de la ciudad de Loja, tomando como base los datos registrados en el Ministerio de Educación hasta diciembre 2018, el número de colegios públicos o fiscales es de 16.

Cabe mencionar que la población a investigar es finita por lo que se procedió a utilizar el muestreo no probabilístico intencional donde el estudio de los elementos es escogido bajo las razones del investigador, tomando en cuenta que la población en su totalidad es de 16 colegios públicos en la ciudad de Loja.

\section{Resultados}

A continuación, se presenta el análisis e interpretación de los resultados obtenidos en las encuestas aplicadas a directores de los colegios públicos o fiscales de la ciudad de Loja, con el afán de obtener la información necesaria que sustente la presente investigación.

Para lo cual, se procedió con el análisis de los datos, para este procedimiento se utilizó herramientas estadísticas, a través de tablas y gráficos estadísticos representativos para el análisis y la interpretación estadística de los resultados; donde a partir de los resultados obtenidos se pudo evidenciar la opinión de los directivos sobre el uso de las técnicas de la información y la comunicación en los colegios públicos de la ciudad de Loja están implementando a los alumnos.

\section{Análisis e interpretación de las encuestas aplicadas a los directores de los colegios públicos} de la ciudad de Loja

\section{1. ¿El colegio al que usted dirige cuenta con un laboratorio de cómputo?}




\section{Tabla 1.}

Laboratorio de cómputo

\begin{tabular}{|c|c|c|}
\hline Descripción & Frecuencia & Porcentaje \\
\hline $\mathrm{Si}$ & 16 & $100 \%$ \\
\hline No & 0 & $0 \%$ \\
\hline Total & 16 & $100 \%$ \\
\hline
\end{tabular}

Fuente: Encuesta a los directores

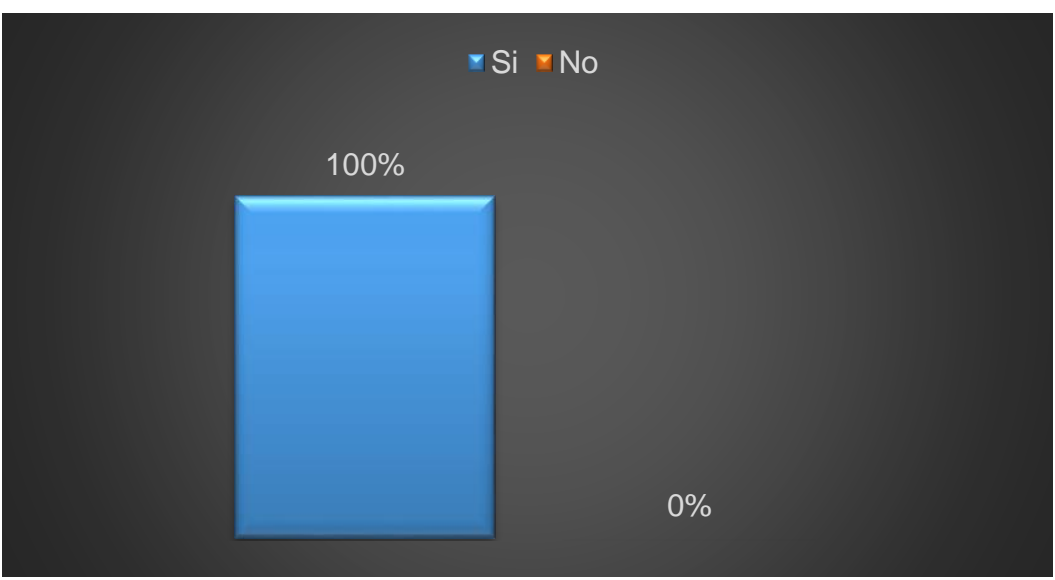

Figura 6. Laboratorio de cómputo

Fuente: Tabla 1.

Análisis e interpretación: El 100\% de los docentes encuestados manifiestan que los colegios cuentan con un laboratorio de cómputo, lo que es importante para el uso y propuesta de las técnicas de información y comunicación ya que permitirá mejora el proceso de enseñanza aprendizaje en los mismos. Por tanto, el departamento de cómputo integra los recursos técnicos y tecnológicos disponibles en un centro educativo para potenciar el proceso de aprendizaje de estudiantes y promover el espíritu de cooperación conjuntamente con los docentes para alcanzar individuos con deseos de investigación, reflexivos, analíticos, críticos y comprometidos con la sociedad y el medio ambiente.

\section{2. ¿Considera que el uso de las técnicas de información permiten adquirir aprendizajes significativos?}

\section{Tabla 2.}

Uso de las técnicas de información

\begin{tabular}{lcc}
\hline Descripción & Frecuencia & Porcentaje \\
\hline $\mathrm{Si}$ & 13 & $81 \%$ \\
$\mathrm{No}$ & 3 & $19 \%$ \\
Total & $\mathbf{1 6}$ & $\mathbf{1 0 0 \%}$ \\
\hline
\end{tabular}

Fuente: Encuesta a los directores 


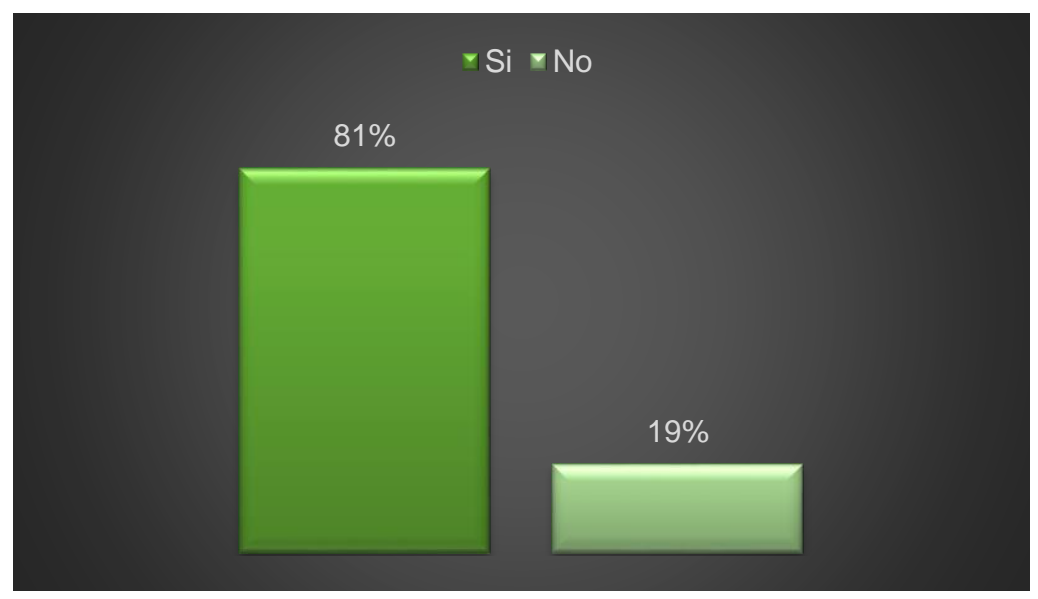

Figura 7. Uso de las técnicas de información

Fuente: Tabla 2.

Análisis e interpretación: El 81\% de los docentes encuestados consideran que el uso de las técnicas de información permiten adquirir aprendizajes significativos, mientras que un 19\% indican que no, esta información nos demuestra que la mayoría de docentes tienen una buena predisposición para utilizar las técnicas de información, el uso de las técnicas de información en la educación aporta más ventajas que desventajas. Porque en la educación tradicional viene a ser un apoyo en demostraciones, dramatizaciones a través de videos, películas y audio casetes. Es decir, se aprende más al utilizar más sentidos en clase que solo estar escuchando al maestro como orador todo el tiempo, motiva la discusión en grupo convirtiendo la clase más interactiva e incluso hay una mayor presentación estética.

\section{3. ¿Cree que la enseñanza virtual favorece la flexibilidad del pensamiento crítico de los estudiantes?}

\section{Tabla 3.}

Enseñanza virtual

\begin{tabular}{|c|c|c|}
\hline Descripción & Frecuencia & Porcentaje \\
\hline $\mathrm{Si}$ & 12 & $75 \%$ \\
\hline No & 4 & $25 \%$ \\
\hline Total & 16 & $100 \%$ \\
\hline
\end{tabular}

Fuente: Encuesta a los directores 


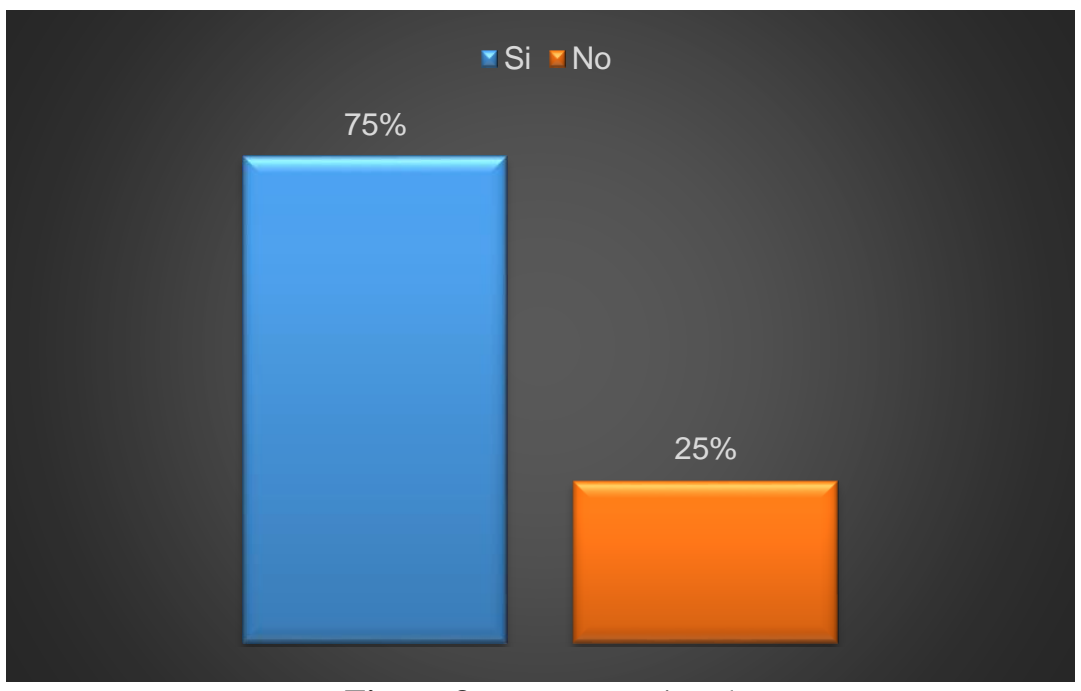

Figura 8. Enseñanza virtual

Fuente: Tabla 3.

Análisis e interpretación: E1 75\% de los docentes consideran que la enseñanza virtual favorece la flexibilidad del pensamiento crítico, mientras que el $25 \%$ indican que no, esta información nos demuestra que la mayoría de docentes tienen una buena predisposición para utilizar la enseñanza virtual. Esta respuesta es importantes ya que los alumnos a través de esta enseñanza virtual tienen disponibilidad de horario las 24 horas al día, los 7 días a la semana, cada estudiante puede establecer su propio horario según las diferentes actividades que realiza, permitiendo aprender cuando quiera y en lugar que quiera evitando la rigidez de tiempo y espacio, además de esta forma los estudiantes ya no se limitan a recibir información del profesor, sino también ellos son los responsables de la construcción de los conocimientos que adquieren, ya que marcan su propio ritmo de aprendizaje.

\section{4. ¿Considera importante la implementación de un aula virtual para el desarrollo del proceso de enseñanza aprendizaje?}

\section{Tabla 4.}

Aula virtual

\begin{tabular}{lcc}
\hline Descripción & Frecuencia & Porcentaje \\
\hline $\mathrm{Si}$ & 15 & $94 \%$ \\
$\mathrm{No}$ & 1 & $6 \%$ \\
Total & $\mathbf{1 6}$ & $\mathbf{1 0 0 \%}$ \\
\hline
\end{tabular}

Fuente: Encuesta a los directores 


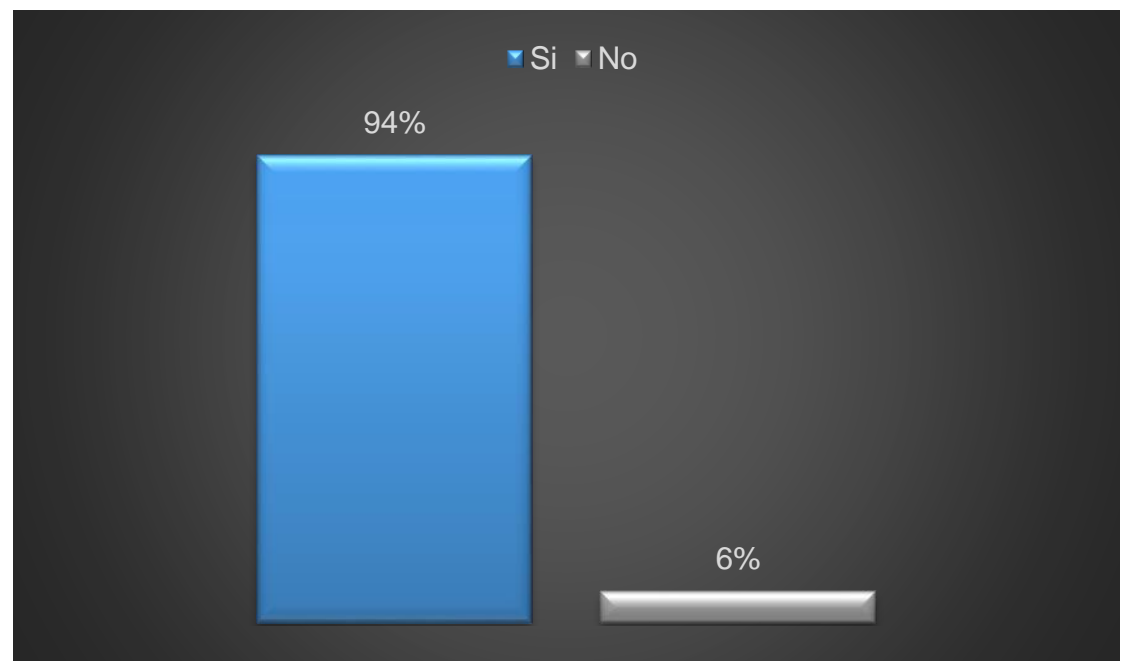

Figura 9. Aula virtual

Fuente: Tabla 4.

Análisis e interpretación: El 94\% de los docentes encuestados consideran importante la implementación de un aula virtual para el desarrollo del proceso de enseñanza aprendizaje, mientras que en un menor porcentaje el $6 \%$ expresan que no. De acuerdo a estos resultados se evidencia que las aulas virtuales debidamente concebidas aportan positivamente, ya que permiten un buen nivel de interacción entre todos los participantes, lo que favorece a los estudiantes para que puedan exponer sus criterios, intercambiar experiencias, trabajar de forma colaborativa, opinar sobre la evaluación de sus compañeros y sobre la suya propia, tener acceso a recursos de diferentes tipos que de otra forma sería prácticamente imposible.

\section{5. ¿Cree que las técnicas de comunicación se han convertido en una poderosa y versátil herramienta que transforma el proceso de enseñanza aprendizaje?}

\section{Tabla 5.}

Técnicas de comunicación

\begin{tabular}{|c|c|c|}
\hline Descripción & Frecuencia & Porcentaje \\
\hline $\mathrm{Si}$ & 16 & $100 \%$ \\
\hline No & 6 & $0 \%$ \\
\hline Total & 16 & $100 \%$ \\
\hline
\end{tabular}




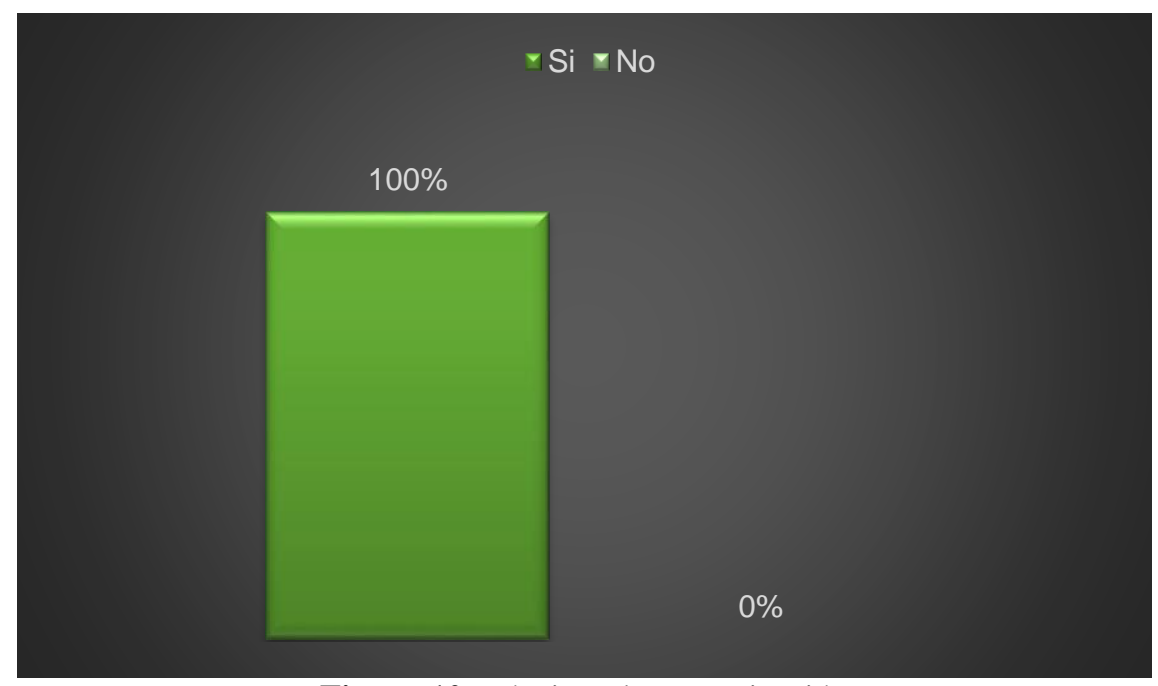

Figura 10. Técnicas de comunicación

Fuente: Tabla 5.

Análisis e interpretación: El 100\% de los docentes consideran que las técnicas de comunicación se han convertido en una poderosa y versátil herramienta que transforma el proceso de enseñanza aprendizaje, se hace necesario apuntar que como nuevo espacio educativo se han convertido en un medio muy utilizado en la educación, a través de ellas docentes y alumnos más allá de las barreras del tiempo y el espacio hacen uso de las computadoras para acceder, comunicar, compartir, crear conocimientos mediante actividades que conducen al aprendizaje.

\section{Discusión}

Los docentes que laboran en el bachillerato en los colegios públicos de la ciudad de Loja, están conformados por profesionales destacándose su formación de 4to nivel, lo que evidencia su preocupación por estar preparados de la mejor manera para enfrentar la ardua labor de formar a sus estudiantes.

Destaca también la combinación de juventud y experiencia lograda, lo cual favorecería para emprender en nuevos proyectos, como por ejemplo el uso de TICS, que se facilita con la presencia de nuevas generaciones de docentes, para quienes es más fácil adaptarse, y mejorar de esta manera los niveles de interactividad; según el autor Peralta (2002) define a las TICS como aquellas herramientas computacionales e informáticas que procesan, sintetizan, recuperan y presentan información para dar forma, registrar, almacenar y difundir contenidos digitalizados.

Por otra parte, es muy satisfactorio verificar que la totalidad de maestros tienen acceso a internet en sus domicilios, y la frecuencia de navegación en internet es aceptable, lo que hace suponer una gran oportunidad para potenciar el uso de herramientas como el uso de un aula virtual, por parte de los docentes. Por tanto, según lo mencionan los autores Fabres, Libuy \& Tapia (2014) el internet es una herramienta de uso masivo que permite a las personas acceder al conocimiento e información de forma casi inmediata y a la carta de quien busque, así, se transforma en una fuente inagotable de datos e información. 
En su totalidad los docentes manifiestan que los colegios cuentan con laboratorios de cómputo y que todos los estudiantes tienen acceso al centro de cómputo, lo que es importante para el uso y propuesta de las técnicas de información y comunicación lo que permitirá mejora el proceso de enseñanza aprendizaje en los alumnos de los colegios públicos de la ciudad de Loja. Los docentes consideran que las técnicas de información permiten adquirir aprendizajes significativos, es decir, la mayoría de docentes tienen una buena predisposición para utilizar las técnicas de información.

Finalmente, los docentes consideran que la enseñanza virtual favorece la flexibilidad del pensamiento crítico, en este sentido consideran importante la implementación de un aula virtual para el desarrollo del proceso de enseñanza aprendizaje ya que las aulas virtuales debidamente concebidas permiten un buen nivel de interacción entre todos los participantes que favorece a los estudiantes pues puedan exponer sus criterios, intercambiar experiencias, trabajar de forma colaborativa, opinar sobre la evaluación de sus compañeros y sobre la suya propia, tener acceso a recursos de diferentes tipos que de otra forma sería prácticamente imposible.

En este sentido, es importante hacer relación con lo que manifiestan los autores Fernández \& Cebreiro (2003) en cuanto a los beneficios que ha traído consigo el ingreso de las TICS en los procesos de enseñanza para favorecer la labor educativa poniendo a disposición de profesores y estudiantes gran cantidad de información a través de diversos canales que favorecen el trabajo colaborativo a través de entornos virtuales permitiendo la comunicación e interacción entre varios usuarios, y el fortalecimiento de destrezas y habilidades en el proceso de aprendizaje.

\section{Conclusiones}

El uso de las técnicas de la información y la comunicación en el ámbito educativo permiten optimizar el manejo de la información y el desarrollo de la comunicación, generando de esta manera un mayor conocimiento e inteligencia.

En los colegios públicos de la ciudad de Loja el uso de las técnicas de la información y la comunicación se ha convertido en una poderosa herramienta para el proceso de enseñanza aprendizaje, ya que es un medio muy utilizado tanto en la educación media como en todos los ámbitos educativos.

Por lo que se recomienda a los docentes y estudiantes hacer un uso adecuado de estas técnicas para acceder, comunicar, compartir y crear conocimientos: ello demuestra principalmente que la utilización de las técnicas de comunicación abre la posibilidad de aumentar el nivel de participación, la comunicación y la socialización del conocimiento con el afán de lograr aprendizajes significativos por medio del uso de recursos tecnológicos.

El presente artículo contribuye a determinar la necesidad e importancia del uso de las TICS como un elemento tecnológico necesario en los colegios públicos de la ciudad de Loja, ya que si se aplican correctamente tiene muchas ventajas como mejora de las herramientas, elementos, habilidades, conocimientos y destrezas que desarrollan los estudiantes al establecer proyectos de manera virtual o en línea. Es decir, cuando la tecnología se aplica en la enseñanza y 
aprendizaje posibilita grandes oportunidades para generar nuevos ambientes de aprendizaje donde los alumnos siendo los entes principales logran aprendizajes significativos acordes al medio en que se desenvuelven.

\section{Bibliografía}

Bautista, A., \& Alba, C. (1997). ¿Qué es tecnología educativa?: Autores y significados. Revista Píxel-bit, $N^{\circ} 9,4$.

Dussel, I., \& Quevedo, L. (2010). Educación y nuevas tecnologías: los desafíos pedagógicos ante el mundo digital. Buenos Aires, República Argentina: Fundación Santillana.

Fabres, B., Libuy, M. D., \& Tapia, G. P. (2014). Análisis del uso de las tecnologías de la información y la comunicación en los establecimientos educacionales de Chile: caso del colegio Santo Tomás de la comuna de ñuñoa. Satiago de Chile: Universidad de Chile.

Fernández, M., \& Cebreiro, B. (2003). La integración de los medios y nuevas tecnologías en los centros y prácticas docentes. Revista Píxel BIT, N(20).

Hilarión, C. L. (2014). Factores que influyen en la no incorporación de las TIC en los procesos de enseñanza - aprendizaje por parte de los docentes en la educación superior. Bogotá: Universidad Militar Nueva Granada.

Jaramillo, L. S., \& González, C. C. (2016). Lás técnicas de la información y comunicación TICS como estrategia metodológica para mejorar el proceso de enseñanza aprendizaje de los alumnos de la especialidad de contabilidad computarizada del Colegio Experimental "Pío Jaramillo Alvarado período 2011". Loja, Ecuador: Universidad Nacional de Loja.

Moreno, T. (2011). Didáctica de la Educación Superior: nuevos desafíos en el siglo XXI. Revista Perspectiva Educacional Vol. 50, № 2. México, 43.

Nieto, M. (2006). Tecnologías de la información y de la comunicación en la educación . Revista Mexicana de Investigación Educativa, 7-10.

Peralta, M. (2002). Una pedagogía de las oportunidades nuevas ventanas para los párvulos del siglo XXI. Chile: Edit. Andrés Bello.

Pérez, J. (2002). Elaboración de un modelo de plataforma digital para el aprendizaje y la generación de conocimientos. Madrid, España: Tesis doctoral Universidad Complutense de Madrid Facultad de Educación.

Ramírez, J. (2009). Tecnologías de la comunicación y la información en cuatro países latinoamericanos. México: Ramírez.

Ramos, V.-G. N. (2015). Uso de las TIC y proceso de enseñanza en la institución educativa numero dos en Maicao-La Guajira-Colombia. Lima-Perú: Universidad Norbert Wiener.

Samillán, L. (06 de 04 de 2010). http://www.scribd.com/doc/3285023/Tics-En - La-Informática. Recuperado el 01 de 2016, de http://www.scribd.com/doc/3285023/TICS-En - LaInformatica: http://www.scribd.com/doc/3285023/TICS-En - La-Informatica

Severin, E. (2010). Tecnologías de la Información y la Comunicación (TIC's) en Educación. España: Banco Interamericano de Desarrollo.

Vera, C. G. (11 de julio de 2012). vinculando.org. Obtenido de TIC's en el proceso de enseñanzaaprendizaje del Castellano: http://vinculando.org/educacion/introduccion-tic-procesoensenanza-aprendizaje-lengua-castellana.html 